\title{
Dual effects of presynaptic membrane mimetics on $\alpha$-synuclein amyloid aggregation
}

\author{
Yuxi Lin ${ }^{1,2 *}$, Dai Ito ${ }^{3}$, Je Min Yoo ${ }^{4}$, Mi Hee Lim ${ }^{5}$, Woo Kyung Yü, Yasushi Kawata7, Young-

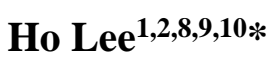

${ }^{1}$ Research Center of Bioconvergence Analysis, Korea Basic Science Institute (KBSI), Ochang, Chungbuk 28119, Republic of Korea

${ }^{2}$ Institute for Protein Research, Osaka University, Yamadaoka 3-2, Suita, Osaka 565-0871, Japan

${ }^{3}$ Department of Brain and Cognitive Science, Daegu Gyeongbuk Institute of Science and Technology (DGIST), Daegu, 42988, Republic of Korea

${ }^{4}$ BIOGRAPHENE, 555 W. $5^{\text {th }}$ St., Los Angeles, California 90013, United States.

${ }^{5}$ Department of Chemistry, Korea Advanced Institute of Science and Technology (KAIST), Daejeon 34141, Republic of Korea

${ }^{6}$ Core Protein Resources Center, Daegu Gyeongbuk Institute of Science and Technology (DGIST), Daegu, 42988, Republic of Korea

${ }^{7}$ Department of Chemistry and Biotechnology, Graduate School of Engineering, Tottori University, Tottori 680-8550, Japan

${ }^{8}$ Bio-Analytical Science, University of Science and Technology (UST), Daejeon 34113, Republic of Korea

${ }^{9}$ Graduate School of Analytical Science and Technology (GRAST), Chungnam National University (CNU), Daejeon 34134, Republic of Korea

${ }^{10}$ Research Headquarters, Korea Brain Research Institute (KBRI), Daegu 41068, Republic of Korea

* Correspondence:

Young-Ho Lee

mr0505@kbsi.re.kr

Yuxi Lin

linyuxi@kbsi.re.kr

Keywords: amyloid fibril, $\alpha$-synuclein, electrostatic interaction, helical structure, intermolecular interaction, membrane mimetic, Parkinson's disease, presynaptic vesicle 


\begin{abstract}
Aggregation of intrinsically disordered $\alpha$-synuclein $(\alpha \mathrm{SN})$ under various conditions is closely related to synucleinopathies. Although various biological membranes have shown to alter the structure and aggregation propensity of $\alpha \mathrm{SN}$, a thorough understanding of the molecular and mechanical mechanism of amyloidogenesis in membranes remains unanswered. Herein, we examined the structural changes, binding properties, and amyloidogenicity of three variations of $\alpha \mathrm{SN}$ mutants under two types of liposomes, 1,2-dioleoyl-sn-glycero-3-phosphocholine (DOPC) and presynaptic vesicle mimetic (Mimic) membranes. While neutrally charged DOPC membranes elicited marginal changes in the structure and amyloid fibrillation of $\alpha$ SNs, negatively charged Mimic membranes induced dramatic helical folding and biphasic amyloid generation. At low concentration of Mimic membranes, the amyloid fibrillation of $\alpha \mathrm{SNs}$ was promoted in a dose-dependent manner. However, further increases in the concentration constrained the fibrillation process. These results suggest the dual effect of Mimic membranes on regulating the amyloidogenesis of $\alpha \mathrm{SN}$, which is rationalized by the amyloidogenic structure of $\alpha \mathrm{SN}$ and condensation-dilution of local $\alpha \mathrm{SN}$ concentration. Finally, we propose physicochemical properties of $\alpha \mathrm{SN}$ and membrane surfaces, and their propensity to drive electrostatic interactions as decisive factors of amyloidogenesis.
\end{abstract}




\section{Introduction}

$\alpha$-Synuclein $(\alpha \mathrm{SN})$, an intrinsically disordered protein consisting of 140 amino acids is abundantly expressed in the brain. Although the exact function of $\alpha \mathrm{SN}$ remains unclear, recent studies suggest that it plays an important role in modulating the neurotransmitter release (Burre, 2015) and protecting nerve terminals (Chandra et al., 2005). However, when exposed to stress conditions such as high levels of reactive oxygen species, soluble $\alpha \mathrm{SN}$ monomers aggregates into insoluble amyloid fibrils with highlyordered cross- $\beta$ structures (Scudamore and Ciossek, 2018). Other forms of aggregates including oligomers are also observed as an intermediate in the process of amyloid fibrillation or as a dead-end product. The abnormal in vivo accumulation of $\alpha \mathrm{SN}$ is the pathological hallmark of synucleinopathies including Parkinson's disease (PD), dementia with Lewy bodies, and multiple system atrophy (MSA).

The self-assembly of $\alpha \mathrm{SN}$ into amyloid fibrils is characterized by two sequential steps: slow nucleation followed by rapid elongation. It is generally accepted that physicochemical and biological factors exert significant impacts on the aggregation kinetics and pathways of $\alpha \mathrm{SN}$. Namely, previous studies indicate that lagged amyloid fibril formation under physiological conditions can be accelerated by increasing temperature to $57^{\circ} \mathrm{C}$ or decreasing $\mathrm{pH}$ to 2.0 (Uversky et al., 2001). The presence of preformed amyloid seeds of lysozyme and insulin also promotes amyloidogenesis of $\alpha \mathrm{SN}$ (Yagi et al., 2005). On the other hand, graphene quantum dots (GQDs), a promising carbon-based nanomaterial in biomedicine, prevent the aggregation of $\alpha \mathrm{SN}$ monomers to amyloids (Kim and Yoo et al., 2018). In addition to $\alpha \mathrm{SN}$, amyloid beta $(\mathrm{A} \beta)$ and tau also display context-dependent aggregation behaviors (Lin et al., 2019; Gee and Lin et al., 2020).

Despite highlighted expression patterns in presynaptic terminals, $\alpha \mathrm{SN}$ is widely distributed in the intracellular environment and interacts with various subcellular components. Among them, lipid membranes have been increasingly accentuated due to their critical impact on the structure and aggregation propensity of $\alpha \mathrm{SN}$. Upon binding to lipid membranes, the amphipathic N-terminal region (NTR) (residues 1 - 60) and the hydrophobic non-amyloid $\beta$ component (NAC) domain (residues $\sim 60$ - 100) adopt $\alpha$-helical structures (Dikiy and Eliezer, 2012). Based on the atomic level analysis, they manifest in two different helical conformations, broken helix and single elongated helix (Pfefferkorn et al., 2012). The distinct structures of $\alpha \mathrm{SN}$ can be attributed to distinctive intermolecular interactions with membranes, which, in turn, dictate the amyloidogenicity of $\alpha \mathrm{SN}$. Along the same lines, the ratio of lipids to proteins (Galvagnion et al., 2015) and other chemical properties of membranes including the charge of head groups (Galvagnion et al., 2016) collectively influence the structure and 
amyloidogenesis of $\alpha \mathrm{SN}$. Moreover, our recent studies revealed that helical conformations in the initial structures of $\alpha \mathrm{SN}$ in membranes is key to amyloid formation (Terakawa, Lee, and Kinoshita et al., 2018a).

Mutations in amyloid precursors are also crucial for regulating amyloidogenicity. For $\alpha \mathrm{SN}$, A53T and H50Q are the representative familial mutants associated with the early onset of PD, which manifest distinct aggregation behaviors and kinetics (Flagmeier et al., 2016; Meade et al., 2019). Truncated forms of $\alpha \mathrm{SN}$ are also observed in Lewy bodies in cells where a truncation at the C-terminal leads to accelerated amyloid formation (Li et al., 2005;Izawa et al., 2012; Sorrentino et al., 2018). Other reports investigate the function of highly acidic $\mathrm{C}$-terminal regions of $\alpha \mathrm{SN}$ in membrane binding and subsequent amyloid formation. Even upon binding to membranes, the C-terminal domain remains disordered by making only weak and transient contacts with membrane surfaces (Fusco et al., 2014). Interestingly, the removal of the C-terminal regions remarkably reshapes the kinetic factors of the aggregation propensity under membrane environments. Although recent advances in characterization techniques have promoted our understanding of the effects of biological membranes on the aggregation of $\alpha \mathrm{SN}$, much remains uncertain about the molecular and mechanical mechanisms of amyloidogenesis of $\alpha \mathrm{SN}$ in membranes.

Herein, we investigated mainly the impacts of presynaptic vesicle-mimicking model (Mimic) membranes on the amyloid fibrillation of $\alpha \mathrm{SN}$. Collective results from the structural change, membrane binding, and amyloid fibrillation of three $\alpha \mathrm{SN}$ variants demonstrated that negatively charged Mimic membranes induce biphasic modulation of the amyloidogenicity of $\alpha \mathrm{SN}$. To explain this dual effect, i.e., promotion and inhibition, we propose two mechanisms based on the amyloidogenic structure of $\alpha \mathrm{SN}$ and the condensation-dilution of local $\alpha \mathrm{SN}$ concentration in membranes. Taken together, this study establishes a general mechanistic perspective on the amyloid fibrillation of $\alpha \mathrm{SN}$ in membranes and thereby contributes to the rational design of candidates against its deleterious aggregation.

\section{Materials and Methods}

\section{Materials}

The full-length human $\alpha \mathrm{SN}$ ( $\alpha \mathrm{SNwT}$ ) and three variations of $\alpha \mathrm{SN}$ mutants: 1) C-terminal 11-residue truncation $\left.\left(\alpha \mathrm{SN}_{129}\right) ; 2\right)$ charge neutralization of negatively-charged residues between positions 130 and 140 to asparagine residues $\left.\left(\alpha \mathrm{SN}_{130 \mathrm{CF}}\right) ; 3\right)$ mutation of the $53^{\text {rd }}$ residue from alanine to threonine 
$\left(\alpha \mathrm{SN}_{\mathrm{A} 53 \mathrm{~T}}\right)$, were expressed in E. coli BL21 (DE3), and purified as previously described (Izawa et al., 2012). Phospholipids, DOPC, 1,2-dioleoyl-sn-glycero-3-phosphoethanolamine (DOPE), and 1,2dioleoyl-sn-glycero-3-phospho-1-serine (DOPS) were obtained from Avanti Polar Lipids Inc. (Alabaster, USA) (Fig. S1). Thioflavin T (ThT) was purchased from Wako Pure Chemical Industries, Ltd (Osaka, Japan). All other reagents were obtained from Nacalai Tesque (Kyoto, Japan).

\section{Vesicle preparation}

Small unilamellar vesicles (SUVs) containing DOPC or DOPC:DOPE:DOPS at a ratio of 2:5:3 were prepared as mimicking presynaptic vesicles according to the previous literature (Terakawa, Lee, and Kinoshita et al., 2018a). Briefly, lipids were dissolved in chloroform, and mixed in glass tubes at the desired compositions. The resulting solution was dried under a nitrogen stream, followed by vacuum drying to ensure the removal of residual organic solvents. To rehydrate the resultant lipid film, a solution of $20 \mathrm{mM}$ sodium phosphate buffer ( $\mathrm{pH}$ 7.4) containing $100 \mathrm{mM} \mathrm{NaCl}$ was added with vortex mixing. After 10 freeze-thaw cycles, lipid suspensions were sonicated for 10 mins on ice to obtain a homogeneous SUVs solution.

\section{ThT fluorescence assay}

$\alpha$ SNs were dissolved in $20 \mathrm{mM}$ sodium phosphate buffer ( $\mathrm{pH} 7.4)$ containing $100 \mathrm{mM} \mathrm{NaCl}$ to prepare a stock concentration of $200 \mu \mathrm{M}$. Protein concentrations were determined using the UV-absorbance at $280 \mathrm{~nm}$ with molar extinction coefficients of $2,980 \mathrm{M}^{-1} \cdot \mathrm{cm}^{-1}$ for $\alpha \mathrm{SN}_{129}$, and 5,960 $\mathrm{M}^{-1} \cdot \mathrm{cm}^{-1}$ for $\alpha \mathrm{SN}_{\mathrm{WT}}$, $\alpha \mathrm{SN}_{130 \mathrm{CF}}$, and $\alpha \mathrm{SN}_{\mathrm{A53T}}$. The following experimental conditions were used to investigate $\alpha \mathrm{SNs}$ amyloid formation at $37{ }^{\circ} \mathrm{C}: 50 \mu \mathrm{M} \alpha \mathrm{SNs}, 20 \mathrm{mM}$ sodium phosphate buffer (pH 7.4), $100 \mathrm{mM} \mathrm{NaCl}, 5 \mu \mathrm{M}$ ThT, and Mimic and DOPC model membranes at various concentrations of lipids. Sample solutions $(200 \mu \mathrm{l})$ were applied in triplicate to each well of the 96-well microplate (Greiner-Bio-One, Tokyo, Japan), and sealed with a film (PowerSeal CRISTAl VIEW, Greiner-Bio-One, Tokyo, Japan). The microplate, placed on a water bath-type ultrasonic transmitter (Elestein SP070- PG-M, Elekon Sci. Inc., Chiba, Japan), was subjected to cycles of ultrasonication for 1 min at 9-min intervals. The fluorescence intensity of ThT was hourly recorded on an SH-9000 microplate reader (Corona Electric Co., Ibaraki, Japan) with excitation and emission wavelengths of 450 and $485 \mathrm{~nm}$, respectively.

After the data acquisition, kinetic analyses of $\alpha \mathrm{SNs}$ amyloid formation were carried out using the following equation: 


$$
Y=y_{i}+m_{\mathrm{i}} t+\frac{y_{\mathrm{f}}+m_{\mathrm{f}} t}{1+\exp \left[-k\left(t-t_{0}\right)\right]}
$$

where $y_{\mathrm{i}}+m_{\mathrm{i}} t$ and $y_{\mathrm{f}}+m_{\mathrm{f}} t$ are the initial and final baselines, respectively. $t_{0}$ is the half-time at which ThT fluorescence reaches $50 \%$ of the maximum amplitude. $k$ represents the elongation rate constant. The lag time was obtained based on the following relationship: lag time $=t_{0}-2(1 / k)$.

\section{Isothermal titration calorimetry}

Isothermal titration calorimetry (ITC) experiments were performed with an ITC 200 instrument (Malvern Panalytical, UK) at $25{ }^{\circ} \mathrm{C}$. The concentrations of $\alpha \mathrm{SN}$ in the ITC syringe and lipids of Mimic membranes in the ITC cell were $400 \mu \mathrm{M}$ and $2 \mathrm{mM}$, respectively. $\alpha$ SNs were dissolved in $20 \mathrm{mM}$ sodium phosphate buffer ( $\mathrm{pH}$ 7.4) containing $100 \mathrm{mM} \mathrm{NaCl}$. The reference power was set to 10 $\mu \mathrm{cal} \cdot \mathrm{sec}^{-1}$, and the initial delay was $300 \mathrm{secs}$. Titration experiments consisted of 20 injections spaced at intervals of $300 \mathrm{secs}$. The injection volume was $0.4 \mu \mathrm{l}$ for the first injection and $2 \mu \mathrm{l}$ for the residual injections. The stirring speed was 1,000 rpm. Data were analyzed with a one-set of sites binding model using the MicroCal PEAQ-ITC Analysis Software (Malvern Panalytical, UK).

\section{Results}

\section{Structural characterization of $\alpha \mathrm{SN}$ mutants under membrane environments}

Far-UV circular dichroism (CD) spectroscopy elucidates the effects of Mimic and DOPC membranes on the initial structures of three different $\alpha \mathrm{SN}$ variants $-\alpha \mathrm{SN}_{129}, \alpha \mathrm{SN}_{130 \mathrm{CF}}$, and $\alpha \mathrm{SN}_{\mathrm{A} 53 \mathrm{~T}}$ (Fig. 1A, E, and I). In the absence of membranes, $\alpha \mathrm{SN}_{129}$ exhibited a single negative band at $200 \mathrm{~nm}$ without any noticeable band in the region between 210 and $230 \mathrm{~nm}$, indicating that the secondary structures are predominantly disordered. On the other hand, increasing the concentration of Mimic lipids from 0 to 5 $\mathrm{mM}$ induced helix-rich conformations as characterized by the two negative bands at $\sim 208$ and $\sim 222$ nm (Fig. 1A, left). Further secondary structure analysis showed consistent results with increased helical structures and decreased $\beta$ - and random-coil structures as a function of Mimic lipids concentration (Fig. S2).

In contrast to Mimic membranes, DOPC membranes caused negligible intensity magnifications in the negative peaks of CD spectra. Even after increasing the concentration of DOPC lipids to $5 \mathrm{mM}$, a minor structural alteration of $\alpha \mathrm{SN}_{129}$ upon binding was still elicited (Fig. 1A, right). Similar structural 
reconfigurations to those of $\alpha \mathrm{SN}_{129}$ were also observed for $\alpha \mathrm{SN}_{130 \mathrm{CF}}$ and $\alpha \mathrm{SN}_{\mathrm{A} 53 \mathrm{~T}}$ in the presence of Mimic and DOPC membranes (Fig. 1E and I). These results indicate that Mimic membranes are more effective in generating helical structures of $\alpha \mathrm{SNs}$, which corroborate our previous findings with $\alpha \mathrm{SN}_{\mathrm{WT}}$ (Terakawa, Lee, and Kinoshita et al., 2018a).

\section{Amyloid formation of $\alpha \mathrm{SN}$ mutants under membrane environments}

ThT fluorescence assay examines the aggregation behaviors of the three $\alpha \mathrm{SN}$ mutants in the absence and presence of the test membranes. In the absence of the membranes, the fluorescence intensities of $\alpha \mathrm{SN}_{129}, \alpha \mathrm{SN}_{130 \mathrm{CF}}$, and $\alpha \mathrm{SN}_{\mathrm{A} 53 \mathrm{~T}}$ increased after a lag phase at $\sim 12-, \sim 8-$, and $\sim 7$-hour post-incubation, and reached a plateau at $\sim 20, \sim 13$, and $\sim 10$ hours after incubation, respectively (Fig. 1B, F, and J). These typical sigmoidal growth curves indicate nucleation-dependent amyloid formation, which was also observed for the amyloid fibrillation of $\alpha \mathrm{SN}_{w}$ in the previous result (Terakawa, Lee, and Kinoshita et al., 2018a). In addition, the post-incubation far-UV CD spectra exhibited a single negative band near $218 \mathrm{~nm}$, representing the cross- $\beta$ structure of amyloid fibrils (Fig. 1C, G, and K). Collectively, the amyloid generation of all three $\alpha \mathrm{SN}$ variants was verified in the absence of membranes.

The presence of Mimic membranes led to more dynamic alterations in the amyloidogenicity of $\alpha \mathrm{SN}_{129}$. ThT fluorescence analysis revealed two distinct effects of Mimic membranes on fibrillation kinetics: 1) accelerated amyloid formation at lower concentrations of Mimic lipids (0.5 - $4 \mathrm{mM})$ with a shorter lag time and larger elongation rate constant; 2) constrained amyloid generation at higher concentrations $(5 \mathrm{mM})$ with a more extended lag time and lower elongation rate constant (Figs. 1B and 2A-C, left). Such results correspond to the previous finding on lipid concentration-dependent amyloidogenesis of $\alpha \mathrm{SN}_{w T}$ (Terakawa, Lee, and Kinoshita et al., 2018a). In accordance with the ThT assay results, the far-UV CD spectra at 0.5 - $3 \mathrm{mM}$ and 4 - $5 \mathrm{mM}$ of Mimic lipids revealed, respectively, amyloid fibrils with $\beta$-structures and monomers with predominant helical conformations (Fig. 1C, left). The atomic force microscopy (AFM) image at $5 \mathrm{mM}$ of Mimic lipids further confirmed their inhibitory effects against amyloidogenesis (Fig. 1D, left).

In contrast to Mimic membranes, DOPC membranes exhibited minimal effects on the amyloid fibrillation of $\alpha \mathrm{SN}_{129}$. As shown in Figure 1B (right), similar nucleation-dependent sigmoidal increases in the ThT intensity were observed at all DOPC lipid concentrations $(0.5-5 \mathrm{mM})$. Further kinetic analyses verified that DOPC lipids at the concentration range between 0.5 and $5 \mathrm{mM}$ slightly promoted $\alpha \mathrm{SN}_{129}$ amyloid formation by affecting the lag time (Fig. 2B, right). The far-UV CD spectra of $\alpha \mathrm{SN}_{129}$ 
at all concentrations of DOPC lipids showed the formation of cross- $\beta$-structured amyloid fibrils after incubation (Fig. 1C, right). It was further verified by AFM analysis at $5 \mathrm{mM}$ of DOPC lipids, which exhibited clustered amyloid fibrils (Fig. 1D, right). Similar minimal effects of DOPC membranes were also revealed for $\alpha \mathrm{SN}_{w T}$ aggregation in the previous literature (Terakawa, Lee, and Kinoshita et al., 2018a).

Next, we investigated the effects of Mimic and DOPC membranes on $\alpha \mathrm{SN}_{130 \mathrm{CF}}$ amyloid formation. The addition of $0.5-2 \mathrm{mM}$ of Mimic lipids remarkably accelerated amyloid fibrillation by shortening the lag time from $\sim 8$ to $\sim 4$ hours (Figs. $1 \mathrm{~F}$ and $2 \mathrm{E}$, left). However, increased lipid concentrations $(4-5 \mathrm{mM})$ impeded the fibrillation of $\alpha \mathrm{SN}_{130 \mathrm{CF}}$, leading to no increase in the ThT intensity throughout incubation. Consistent with ThT results, far-UV CD spectra at upper range lipid concentrations showed predominant helical structures (Fig. 1G, left) without detectable fibrillar aggregates at $5 \mathrm{mM}$ of Mimic lipids (Fig. 1H, left). On the other hand, the presence of DOPC membranes did not yield noticeable changes on the amyloid formation of $\alpha \mathrm{SN}_{130 \mathrm{CF}}$. At all concentrations of DOPC lipids, ThT intensities increased after a lag time of $\sim 8-\sim 11$ hours (Figs. 1F and $2 \mathrm{E}$, right). Although the maximum ThT intensities at high concentrations of DOPC lipids were greater than those at low and middle concentrations (Fig. 2D, right), such discrepancy can be attributed to the polymorphic nature of amyloid fibrils. Along the same lines, cross- $\beta$ structures were detected at all DOPC concentrations after incubation (Fig. 1G, right), with evident fibrillar aggregates formation at $5 \mathrm{mM}$ of DOPC (Fig. 1H, right).

Analogous to the findings for $\alpha \mathrm{SN}_{129}$ and $\alpha \mathrm{SN}_{130 \mathrm{CF}}$, Mimic membranes accelerated and inhibited the amyloidogenesis of $\alpha \mathrm{SN}_{\mathrm{A} 53 \mathrm{~T}}$ in a concentration-dependent manner. As the concentration of Mimic lipids increased from 0 to $5 \mathrm{mM}$, the lag time initially decreased and then increased, with the elongation rate constant showing the opposite tendency (Figs. 1J, 2H and I, left). While some fibrillar fragments were observed at $5 \mathrm{mM}$ of Mimic lipids (Fig. 1L, left), the majority of $\alpha \mathrm{SN}_{\mathrm{A} 53 \mathrm{~T}}$ existed as helical monomers (Figs. $1 \mathrm{~K}$ and $2 \mathrm{G}$, left). On the contrary, almost no effect of DOPC membranes on the amyloid formation of $\alpha \mathrm{SN}_{\mathrm{A} 53 \mathrm{~T}}$ was detected. Kinetic analyses of the ThT data revealed that the lag time and elongation rate constant of $\alpha \mathrm{SN}_{\mathrm{A} 53 \mathrm{~T}}$ fibrillation remained steady throughout all lipid concentrations (Fig. 2H and I, right). In like manner, far-UV CD spectra indicated the existence of fibrillar aggregates with cross- $\beta$ structures at all DOPC concentrations (Fig. 1K, right), which was supported by the representative AFM image in the presence of $5 \mathrm{mM}$ of DOPC lipids (Fig. 1L, right). 


\section{Calorimetry-based investigation of intermolecular interactions between Mimic membranes and $\alpha \mathrm{SNs}$}

To obtain further insights into how Mimic membranes modulate the amyloidogenicity of $\alpha \mathrm{SN}$, we performed ITC analysis on $\alpha \mathrm{SN}$-membrane interactions. As shown in Figure 3A-D (upper), a series of titration of $\alpha \mathrm{SNs}$ to Mimic membranes generated negative ITC peaks followed by gradual saturation. This suggests the presence of appreciable exothermal intermolecular interactions between $\alpha$ SNs and Mimic membranes. Following normalization of all ITC peaks, ITC thermograms were converted to binding isotherms (Fig. 3A-D, lower). The thermodynamic parameters obtained from fitting the binding isotherms are summarized in Figure 3E.

As expected from downward ITC peaks, thermodynamically favorable enthalpy changes $(\Delta H<$ 0 ), ranging from $\sim-200$ to $\sim-300 \mathrm{kcal} \cdot \mathrm{mol}^{-1}$, ensued for all variants of $\alpha \mathrm{SN}$. A large negative value of $\Delta H$ may stem from the membrane binding of $\alpha \mathrm{SNs}$ being steered by attractive electrostatic interactions and concomitant helical folding. On one hand, unfavorable negative entropy change $(T \Delta S<0)$ were monitored from -190 to $\sim-290 \mathrm{kcal} \cdot \mathrm{mol}^{-1}$. Nevertheless, the loss of conformational and translational entropies owing to the membrane-induced helical folding of $\alpha \mathrm{SNs}$ and the restricted lipid diffusion were compensated by large negative $\Delta H$ resulting in thermodynamic stabilization. The outcomes demonstrate that $\alpha$ SNs-Mimic membrane interactions are purely driven by the enthalpy change. Similar trends entailed for the interactions between $\alpha \mathrm{SN}_{\mathrm{WT}}$ and model membranes which consisted of phosphatidylserine and gangliosidosis-1 (Nuscher et al., 2004;Bartels et al., 2014).

ITC analyses provided distinct dissociation constant $\left(K_{\mathrm{d}}\right)$ for all binding systems which are within a similar range. The changes in the Gibbs free energy $(\Delta G)$ showed negative values ranging from -9.0 to $-9.4 \mathrm{kcal} \cdot \mathrm{mol}^{-1}$, which indicate spontaneous interactions of all $\alpha \mathrm{SN}$ variants with Mimic membranes. It should be noted that the binding affinity decreased in the order of $\alpha \mathrm{SN}_{130 \mathrm{CF}}, \alpha \mathrm{SN}_{129}, \alpha \mathrm{SN}_{\mathrm{wT}}$, and $\alpha \mathrm{SN}_{\mathrm{A53}}$. Altogether, the findings from the ITC study suggest that the negative charges of the Cterminal region play a pivotal role in the thermodynamic adjustment of $\alpha \mathrm{SN}$ upon binding to Mimic membranes.

\section{Discussion}

We investigated the impacts of lipid membranes on the amyloid formation of three variations of $\alpha \mathrm{SNs}$ $\left(\alpha \mathrm{SN}_{129}, \alpha \mathrm{SN}_{130 \mathrm{CF}}\right.$, and $\left.\alpha \mathrm{SN}_{\mathrm{A53T}}\right)$ with different charge states and lengths as functions of lipid 
component and concentration. Based on the structural, kinetic, and thermodynamic characterizations, the molecular and mechanical mechanisms of membrane-assisted acceleration and inhibition of amyloid generation were elucidated (Figs 1, 2, and S1). While neutrally charged DOPC membranes showed insignificant effects on the structure and amyloidogenicity of $\alpha \mathrm{SNs}$, negatively charged Mimic membranes induced dramatic helical transitions with the dual effects of promoting and impeding amyloid aggregation depending on the membrane concentration. At low concentrations of Mimic lipids, the fibrillation of $\alpha \mathrm{SNs}$ was accelerated, whereas high lipid concentrations abrogated the process. Similar dual effects on the amyloidogenicity of $\alpha \mathrm{SN}_{\mathrm{wT}}$ were reported for other membranes with negatively charged lipids such as DOPS and DMPS (Galvagnion et al., 2015;Galvagnion et al., 2016), as well as SDS (Giehm et al., 2010). Thus, these findings strongly implicate lipid concentrationdependent acceleration and inhibition of $\alpha \mathrm{SN}$ amyloidogenesis under membrane-binding conditions with a net negative charge of the head groups.

To rationalize the dual effects of negatively charged Mimic membranes, we conceive two possible mechanisms based on the initial structure of $\alpha \mathrm{SN}$ in membranes (the amyloidogenic structure) and the intermolecular affinity of $\alpha \mathrm{SN}$ for membranes (condensation-dilution). The initial structure model elucidates the dual effect based on distinct structures of $\alpha$ SNs at varying lipid concentrations (Fig. 4A). In the absence of Mimic lipids, largely disordered $\alpha$ SNs slowly self-assemble into amyloid fibrils with cross- $\beta$ structures. The addition of Mimic lipids at low concentrations triggers structural alteration from random coils to partial helical structures (Fig. 4A, upper). Partial helical structures are inclined to interact with one another through helix-helix interactions, thereby facilitating nucleation for amyloidogenesis (Abedini and Raleigh, 2009; Lin et al., 2019).

Previous studies also suggested that partial helical structures are aggregation-prone and are the representative secondary structures of the key intermediates in the fibrillation pathway of $\alpha \mathrm{SN}$ (Anderson et al., 2010;Ghosh et al., 2015), Aß40 (Lin et al., 2019), hIAPP (Pannuzzo et al., 2013), and polyQ (Jayaraman et al., 2012), proposing an existence of amyloidogenic structure. Amyloidogenic structures have also been implicated in other folded proteins such as SH3 domain (Guijarro et al., 1998) and $\beta 2$-microglobulin (Jahn et al., 2006). In contrast, at high concentrations of Mimic lipids, $\alpha$ SNs adopt prominent helical structures with an exceptionally low aggregation propensity (Fig. 4A, lower). These highly helical non-amyloidogenic structures were analogously observed in $A \beta 40, A \beta 42$, and $\alpha \mathrm{SNs}$ at high concentrations of alcohols (e.g. 40\% TFE and 50\% HFIP) (Crescenzi et al., 
2002; Anderson et al., 2010; Lin et al., 2019). Accordingly, $\alpha$ SNs in bulk aqueous solution would take time to form a nucleus with an amyloidogenic structure in a conformational ensemble.

Condensation-dilution model explains the mechanism of the dual effect on the basis of the thermodynamic binding affinity (Fig. 4B). At low lipid concentrations, $\alpha$ SN binds multiply with Mimic membranes, which leads to increased local concentrations of $\alpha \mathrm{SN}$ (Fig. 4B, left). Thus, concentrated $\alpha \mathrm{SN}$ will be sufficient to facilitate nucleation for amyloid fibrillation. The growth process can be expedited by elongation with the addition of neighboring monomers around fibril seeds. However, at high lipid concentrations, $\alpha$ SNs will be spread across discrete liposomes and their membranes, leading to diluted local concentrations of $\alpha \mathrm{SN}$. As a result, the amount of $\alpha \mathrm{SNs}$ in each liposome and bulk water decrease significantly. This, in turn, interferes with efficient nucleation and elongation, causing the prevention of amyloid formation (Fig. 4B, right). In addition, the condensation-dilution model also illustrates the aggregation of $\mathrm{A} \beta$ at the various concentration of cationic polystyrene nanoparticles (Cabaleiro-Lago et al., 2010).

Biological membranes have shown their capability to modulate folding, aggregation, and the function of $\alpha \mathrm{SN}$ (O'Leary and Lee, 2019). Binding affinity of $\alpha \mathrm{SN}$ for membranes is influenced not only by the properties of lipid bilayers such as the net charge and curvature, but also by mutations and post-translational modifications including phosphorylation (Kuwahara et al., 2012) and N-terminal acetylation (Runfola et al., 2020). In the current study, we revealed that the binding affinity of $\alpha$ SNs to Mimic membranes decreased in the order $\alpha \mathrm{SN}_{130 \mathrm{CF}}, \alpha \mathrm{SN}_{129}, \alpha \mathrm{SN}_{w T}$, and $\alpha \mathrm{SN}_{\mathrm{A} 53 \mathrm{~T}}$. This indicates that the removal of negatively charged residues between positions 130 and 140 increases the membrane binding affinity, whereas repulsive electrostatic interactions between negatively charged C-terminal domain of $\alpha \mathrm{SN}$ and Mimic membranes decrease the intermolecular affinity. Considering that the large energy gain for $\alpha \mathrm{SN}$ upon membrane binding is derived from electrostatic interactions between the positively charged NTR of $\alpha \mathrm{SN}$ and negatively charged membranes, electrostatic forces are fundamental for $\alpha \mathrm{SN}$-membrane interactions. Increased affinity for membranes with an additional positive charge in the NTR of E46K further supports the importance of electrostatic contributions (Stockl et al., 2008). In addition, we speculate that a point mutation in the NTR like $\alpha$ SNA53T might impair favorable electrostatic interactions with membranes, which attenuates the overall affinity.

In line with present results, a recent study reported that the addition of calcium ions significantly increases $\alpha$ SN's propensity to interact with negatively charged membranes by reducing repulsive electrostatic interactions of negatively charged C-terminal regions with membranes (Lautenschlager et 
al., 2018). Along the same lines, the higher affinity of $\alpha \mathrm{SN}_{130 \mathrm{CF}}$ can be attributed to possible contacts of neutralized 10 residues with membranes via non-polar interactions. It should be also noted that the minimal concentration of Mimic lipids for blocking fibrillation $\left(\alpha \mathrm{SN}_{130 \mathrm{CF}}: 4 \mathrm{mM} ; \alpha \mathrm{SN}_{129} \approx \alpha \mathrm{SN}_{\mathrm{wT}}: 5\right.$ $\mathrm{mM}$; $\alpha \mathrm{SN}_{\mathrm{A} 53 \mathrm{~T}}$ : $>5 \mathrm{mM}$ ) mostly followed the reverse order of the binding affinity, which further supports the condensation-dilution model. Overall, the relative molar ratio of $\alpha \mathrm{SN}$ to the lipid concentration is a decisive parameter of amyloid generation in presynaptic vesicles.

Phase diagrams are highly valuable for a comprehensive understanding biological and pathogenic phase transitions including protein aggregation (Lin and Lee et al., 2014;Lin et al., 2016; Terakawa, Lee, and Kinoshita et al., 2018a;Terakawa et al., 2018b;Lin et al., 2019; Gee and Lin et al., 2020; Ivanova et al., 2021). To illustrate membrane-induced amyloidogenesis of $\alpha$ SNs, we constructed conceptual phase diagrams of $\alpha \mathrm{SN}_{130 \mathrm{CF}}, \alpha \mathrm{SN}_{129}$, and $\alpha \mathrm{SN}_{\mathrm{A} 53 \mathrm{~T}}$ depending on the concentrations of $\alpha \mathrm{SN}$ and Mimic lipids (Fig. S3). Each $\alpha \mathrm{SN}$ displays soluble-to-insoluble phase transition following thermodynamic equilibration. Displaying amyloid-forming regions of $\alpha \mathrm{SN}_{130 \mathrm{CF}}$, $\alpha \mathrm{SN}_{129}$, and $\alpha \mathrm{SN}_{\mathrm{A} 53 \mathrm{~T}}$ at $50 \mu \mathrm{M}$ respectively at $0-4,0-3$, and $0-5 \mathrm{mM}$ of Mimic lipids demonstrate the minimal concentration of Mimic lipids required to impede the fibrillation process. Further elevations in the lipid concentration beyond the amyloid-forming region may increase the solubility of $\alpha \mathrm{SNs}$, thereby preventing their aggregation.

Understanding of context-dependent kinetics and amyloidogenicity of $\alpha \mathrm{SN}$ is essential for overcoming synucleinopathies with cytotoxic aggregation in cells. Depending on its cellular localization and neighboring components, the amyloid fibrillation of $\alpha \mathrm{SN}$ will be both faster and slower in bulk solution than in biological membranes, including presynaptic vesicles, due to the dual effect. As the dual effect based on two possible models suggests, $\alpha \mathrm{SN}$ amyloid fibrillation is subjected to acceleration or inhibition depending on the structural state of $\alpha \mathrm{SN}$ and its relative affinity for membranes. In summation, the modulation of amyloidogenesis is governed by various conditions that regulate electrostatic interactions between $\alpha \mathrm{SN}$ and membranes through a favorable enthalpic contribution.

\section{Author Contributions}

Y.L. and Y.-H.L. conceived the presented idea. Y.L. and D.I. carried out the experiment. D.I., M.H.L. J.M.Y., W.K.Y., and Y.K. contributed to the interpretation of the results and edited the manuscript. 
Y.L. and Y.-H.L. wrote the manuscript with input from all authors. All authors contributed to the article and approved the submitted version.

\section{Funding}

This research was supported by and the National Research Foundation of Korea (NRF) grant (NRF2019R1A2C1004954) (to Y.-H.L.) and the KBSI funds (C130000, C180310, and C140130) (to Y.H.L).

\section{Conflict of Interest}

The authors declare that the research was conducted in the absence of any commercial or financial relationships that could be construed as a potential conflict of interest.

\section{Acknowledgments}

We thank Dr. Mayu. S. Terakawa (Kyoto Univ., Japan) and Prof. Yuji Goto (Osaka Univ., Japan) for their help to the current study. We thank Prof. Masahiro Shirakawa (Kyoto Univ., Japan) for contributing to ITC experiments. The authors acknowledge J.P. Hostetler (BIOGRAPHENE, USA) for assisting in manuscript completion and revision.

\section{Reference}

Abedini, A., and Raleigh, D.P. (2009). A role for helical intermediates in amyloid formation by natively unfolded polypeptides? Phys. Biol. 6, 015005. 10.1088/1478-3975/6/1/015005

Anderson, V.L., Ramlall, T.F., Rospigliosi, C.C., Webb, W.W., and Eliezer, D. (2010). Identification of a helical intermediate in trifluoroethanol-induced alpha-synuclein aggregation. Proc. Natl. Acad. Sci. U. S. A. 107, 18850-18855. 10.1073/pnas.1012336107

Bartels, T., Kim, N.C., Luth, E.S., and Selkoe, D.J. (2014). N-alpha-acetylation of alpha-synuclein increases its helical folding propensity, GM1 binding specificity and resistance to aggregation. PLoS One 9, e103727. 10.1371/journal.pone.0103727

Burre, J. (2015). The Synaptic Function of alpha-Synuclein. J. Parkinsons Dis. 5, 699-713. 10.3233/JPD-150642 
Cabaleiro-Lago, C., Quinlan-Pluck, F., Lynch, I., Dawson, K.A., and Linse, S. (2010). Dual effect of amino modified polystyrene nanoparticles on amyloid beta protein fibrillation. ACS Chem. Neurosci. 1, 279-287. 10.1021/cn900027u

Chandra, S., Gallardo, G., Fernandez-Chacon, R., Schluter, O.M., and Sudhof, T.C. (2005). Alphasynuclein cooperates with CSPalpha in preventing neurodegeneration. Cell 123, 383-396. 10.1016/j.cell.2005.09.028

Crescenzi, O., Tomaselli, S., Guerrini, R., Salvadori, S., D'ursi, A.M., Temussi, P.A., and Picone, D. (2002). Solution structure of the Alzheimer amyloid beta-peptide (1-42) in an apolar microenvironment. Similarity with a virus fusion domain. Eur. J. Biochem. 269, 5642-5648. 10.1046/j.14321033.2002.03271.x

Dikiy, I., and Eliezer, D. (2012). Folding and misfolding of alpha-synuclein on membranes. Biochim. Biophys. Acta 1818, 1013-1018. 10.1016/j.bbamem.2011.09.008

Flagmeier, P., Meisl, G., Vendruscolo, M., Knowles, T.P., Dobson, C.M., Buell, A.K., and Galvagnion, C. (2016). Mutations associated with familial Parkinson's disease alter the initiation and amplification steps of alpha-synuclein aggregation. Proc. Natl. Acad. Sci. U. S. A. 113, 10328-10333. 10.1073/pnas. 1604645113

Fusco, G., De Simone, A., Gopinath, T., Vostrikov, V., Vendruscolo, M., Dobson, C.M., and Veglia, G. (2014). Direct observation of the three regions in alpha-synuclein that determine its membranebound behaviour. Nat. Commun. 5, 3827. 10.1038/ncomms4827

Galvagnion, C., Brown, J.W., Ouberai, M.M., Flagmeier, P., Vendruscolo, M., Buell, A.K., Sparr, E., and Dobson, C.M. (2016). Chemical properties of lipids strongly affect the kinetics of the membraneinduced aggregation of alpha-synuclein. Proc. Natl. Acad. Sci. U. S. A. 113, 7065-7070. 10.1073/pnas.1601899113

Galvagnion, C., Buell, A.K., Meisl, G., Michaels, T.C., Vendruscolo, M., Knowles, T.P., and Dobson, C.M. (2015). Lipid vesicles trigger alpha-synuclein aggregation by stimulating primary nucleation. Nat. Chem. Biol. 11, 229-234. 10.1038/nchembio. 1750

Gee, N., Y., L., H., L.M., and Y.-H., L. (2020). Key Physicochemical and Biological Factors of the Phase Behavior of Tau. Chem 6, 2924-2963. 10.1016/j.chempr.2020.09.012

Ghosh, D., Singh, P.K., Sahay, S., Jha, N.N., Jacob, R.S., Sen, S., Kumar, A., Riek, R., and Maji, S.K. (2015). Structure based aggregation studies reveal the presence of helix-rich intermediate during alphaSynuclein aggregation. Sci. Rep. 5, 9228. 10.1038/srep09228 
Giehm, L., Oliveira, C.L., Christiansen, G., Pedersen, J.S., and Otzen, D.E. (2010). SDS-induced fibrillation of alpha-synuclein: an alternative fibrillation pathway. J. Mol. Biol. 401, 115-133. 10.1016/j.jmb.2010.05.060

Guijarro, J.I., Sunde, M., Jones, J.A., Campbell, I.D., and Dobson, C.M. (1998). Amyloid fibril formation by an SH3 domain. Proc. Natl. Acad. Sci. U. S. A. 95, 4224-4228. 10.1073/pnas.95.8.4224 Ivanova, M.I., Lin, Y., Lee, Y.H., Zheng, J., and Ramamoorthy, A. (2021). Biophysical processes underlying cross-seeding in amyloid aggregation and implications in amyloid pathology. Biophys Chem 269, 106507. 10.1016/j.bpc.2020.106507

Izawa, Y., Tateno, H., Kameda, H., Hirakawa, K., Hato, K., Yagi, H., Hongo, K., Mizobata, T., and Kawata, Y. (2012). Role of C-terminal negative charges and tyrosine residues in fibril formation of alpha-synuclein. Brain Behav. 2, 595-605. 10.1002/brb3.86

Jahn, T.R., Parker, M.J., Homans, S.W., and Radford, S.E. (2006). Amyloid formation under physiological conditions proceeds via a native-like folding intermediate. Nat. Struct. Mol. Biol. 13, 195-201. 10.1038/nsmb1058

Jayaraman, M., Kodali, R., Sahoo, B., Thakur, A.K., Mayasundari, A., Mishra, R., Peterson, C.B., and Wetzel, R. (2012). Slow amyloid nucleation via alpha-helix-rich oligomeric intermediates in short polyglutamine-containing huntingtin fragments. J. Mol. Biol. 415, 881-899. 10.1016/j.jmb.2011.12.010

Kim, D., Yoo, J.M., Hwang, H., Lee, J., Lee, S.H., Yun, S.P., Park, M.J., Lee, M., Choi, S., Kwon, S.H., Lee, S., Kwon, S.H., Kim, S., Park, Y.J., Kinoshita, M., Lee, Y.H., Shin, S., Paik, S.R., Lee, S.J., Lee, S., Hong, B.H., and Ko, H.S. (2018). Graphene quantum dots prevent alpha-synucleinopathy in Parkinson's disease. Nat Nanotechnol 13, 812-818. 10.1038/s41565-018-0179-y

Kuwahara, T., Tonegawa, R., Ito, G., Mitani, S., and Iwatsubo, T. (2012). Phosphorylation of alphasynuclein protein at Ser-129 reduces neuronal dysfunction by lowering its membrane binding property in Caenorhabditis elegans. J. Biol. Chem. 287, 7098-7109. 10.1074/jbc.M111.237131

Lautenschlager, J., Stephens, A.D., Fusco, G., Strohl, F., Curry, N., Zacharopoulou, M., Michel, C.H., Laine, R., Nespovitaya, N., Fantham, M., Pinotsi, D., Zago, W., Fraser, P., Tandon, A., St GeorgeHyslop, P., Rees, E., Phillips, J.J., De Simone, A., Kaminski, C.F., and Schierle, G.S.K. (2018). Cterminal calcium binding of alpha-synuclein modulates synaptic vesicle interaction. Nat. Commun. 9, 712. 10.1038/s41467-018-03111-4

Li, W., West, N., Colla, E., Pletnikova, O., Troncoso, J.C., Marsh, L., Dawson, T.M., Jakala, P., Hartmann, T., Price, D.L., and Lee, M.K. (2005). Aggregation promoting C-terminal truncation of 
alpha-synuclein is a normal cellular process and is enhanced by the familial Parkinson's disease-linked mutations. Proc. Natl. Acad. Sci. U. S. A. 102, 2162-2167. 10.1073/pnas.0406976102

Lin, Y., Kardos, J., Imai, M., Ikenoue, T., Kinoshita, M., Sugiki, T., Ishimori, K., Goto, Y., and Lee, Y.H. (2016). Amorphous Aggregation of Cytochrome c with Inherently Low Amyloidogenicity Is Characterized by the Metastability of Supersaturation and the Phase Diagram. Langmuir 32, 20102022. 10.1021/acs.langmuir.5b03810

Lin, Y., Lee, Y.H., Yoshimura, Y., Yagi, H., and Goto, Y. (2014). Solubility and supersaturationdependent protein misfolding revealed by ultrasonication. Langmuir 30, 1845-1854. 10.1021/la403100h

Lin, Y., Sahoo, B.R., Ozawa, D., Kinoshita, M., Kang, J., Lim, M.H., Okumura, M., Huh, Y.H., Moon, E., Jang, J.H., Lee, H.J., Ryu, K.Y., Ham, S., Won, H.S., Ryu, K.S., Sugiki, T., Bang, J.K., Hoe, H.S., Fujiwara, T., Ramamoorthy, A., and Lee, Y.H. (2019). Diverse Structural Conversion and Aggregation Pathways of Alzheimer's Amyloid-beta (1-40). ACS Nano 13, 8766-8783. 10.1021/acsnano.9b01578 Meade, R.M., Fairlie, D.P., and Mason, J.M. (2019). Alpha-synuclein structure and Parkinson's disease - lessons and emerging principles. Mol. Neurodegener. 14, 29. 10.1186/s13024-019-0329-1

Nuscher, B., Kamp, F., Mehnert, T., Odoy, S., Haass, C., Kahle, P.J., and Beyer, K. (2004). Alphasynuclein has a high affinity for packing defects in a bilayer membrane: a thermodynamics study. $J$. Biol. Chem. 279, 21966-21975. 10.1074/jbc.M401076200

O'leary, E.I., and Lee, J.C. (2019). Interplay between alpha-synuclein amyloid formation and membrane structure. Biochim. Biophys. Acta Proteins Proteom. 1867, 483-491. 10.1016/j.bbapap.2018.09.012

Pannuzzo, M., Raudino, A., Milardi, D., La Rosa, C., and Karttunen, M. (2013). alpha-helical structures drive early stages of self-assembly of amyloidogenic amyloid polypeptide aggregate formation in membranes. Sci. Rep. 3, 2781. 10.1038/srep02781

Pfefferkorn, C.M., Jiang, Z., and Lee, J.C. (2012). Biophysics of alpha-synuclein membrane interactions. Biochim. Biophys. Acta 1818, 162-171. 10.1016/j.bbamem.2011.07.032

Runfola, M., De Simone, A., Vendruscolo, M., Dobson, C.M., and Fusco, G. (2020). The N-terminal Acetylation of alpha-Synuclein Changes the Affinity for Lipid Membranes but not the Structural Properties of the Bound State. Sci. Rep. 10, 204. 10.1038/s41598-019-57023-4

Scudamore, O., and Ciossek, T. (2018). Increased Oxidative Stress Exacerbates alpha-Synuclein Aggregation In Vivo. J. Neuropathol. Exp. Neurol. 77, 443-453. 10.1093/jnen/nly024 
Sorrentino, Z.A., Vijayaraghavan, N., Gorion, K.M., Riffe, C.J., Strang, K.H., Caldwell, J., and Giasson, B.I. (2018). Physiological C-terminal truncation of alpha-synuclein potentiates the prion-like formation of pathological inclusions. J. Biol. Chem. 293, 18914-18932. 10.1074/jbc.RA118.005603

Stockl, M., Fischer, P., Wanker, E., and Herrmann, A. (2008). Alpha-synuclein selectively binds to anionic phospholipids embedded in liquid-disordered domains. J. Mol. Biol. 375, 1394-1404. 10.1016/j.jmb.2007.11.051

Terakawa, M.S., Lee, Y.H., Kinoshita, M., Lin, Y., Sugiki, T., Fukui, N., Ikenoue, T., Kawata, Y., and Goto, Y. (2018a). Membrane-induced initial structure of alpha-synuclein control its amyloidogenesis on model membranes. Biochim. Biophys. Acta Biomembr. 1860, 757-766. 10.1016/j.bbamem.2017.12.011

Terakawa, M.S., Lin, Y., Kinoshita, M., Kanemura, S., Itoh, D., Sugiki, T., Okumura, M., Ramamoorthy, A., and Lee, Y.H. (2018b). Impact of membrane curvature on amyloid aggregation. Biochim. Biophys. Acta Biomembr. 1860, 1741-1764. 10.1016/j.bbamem.2018.04.012

Uversky, V.N., Li, J., and Fink, A.L. (2001). Evidence for a partially folded intermediate in alphasynuclein fibril formation. J. Biol. Chem. 276, 10737-10744. 10.1074/jbc.M010907200

Yagi, H., Kusaka, E., Hongo, K., Mizobata, T., and Kawata, Y. (2005). Amyloid fibril formation of alpha-synuclein is accelerated by preformed amyloid seeds of other proteins: implications for the mechanism of transmissible conformational diseases. J. Biol. Chem. 280, 38609-38616. 10.1074/jbc.M508623200 


\section{Figure legends}

Figure 1. Effects of model membranes on the structure and amyloid formation of aSNs. (A-L) Conformational transitions and fibrillation kinetics of $\alpha \mathrm{SN}_{129}(\mathbf{A}-\mathbf{D}), \alpha \mathrm{SN}_{130 \mathrm{CF}}(\mathbf{E}-\mathbf{H})$, and $\alpha \mathrm{SN}_{\mathrm{A} 53 \mathrm{~T}}$ (I$\mathbf{L}$ ) in the absence and presence of Mimic (left) and DOPC membranes (right). Far-UV CD spectra of $\alpha \mathrm{SN}_{129}(\mathbf{A}$ and $\mathbf{C}), \alpha \mathrm{SN}_{130 \mathrm{CF}}(\mathbf{E}$ and $\mathbf{G})$, and $\alpha \mathrm{SN}_{\mathrm{A} 33 \mathrm{~T}}(\mathbf{I}$ and $\mathbf{K})$ before $(\mathbf{A}, \mathbf{E}$, and $\mathbf{I})$ and after $(\mathbf{C}, \mathbf{G}$, and K) incubation were acquired. (B, F, and $\mathbf{J})$ Fibrillation kinetics of $\alpha \mathrm{SN}_{129}(\mathbf{B}), \alpha \mathrm{SN}_{130 \mathrm{CF}}(\mathbf{F})$, and $\alpha \mathrm{SN}_{\mathrm{A53T}}(\mathbf{J})$ were monitored by the ThT fluorescence assay. Raw data averaged from three independent experiments are shown as closed circles. Solid lines represent the fit curves. Schematic representations of $\alpha \mathrm{SN}_{129}, \alpha \mathrm{SN}_{130 \mathrm{CF}}$, and $\alpha \mathrm{SN}_{\mathrm{A} 53 \mathrm{~T}}$ are displayed above the corresponding data. The $N$-terminal region (NTR), the non-amyloid $\beta$ component (NAC) region, and the $C$-terminal region (CTR) are colored in blue, grey, and red, respectively. Various concentrations of lipids in Mimic and DOPC membranes are guided by distinct colors: black $(0 \mathrm{mM})$, light blue $(0.5 \mathrm{mM})$, blue $(1 \mathrm{mM})$, green $(2 \mathrm{mM})$, yellow (3 mM), pink (4 mM), and red $(5 \mathrm{mM})$. (D, H, and $\mathbf{L})$ AFM images were taken for the samples of $\alpha \mathrm{SN}_{129}(\mathbf{D}), \alpha \mathrm{SN}_{130 \mathrm{CF}}(\mathbf{H})$, and $\alpha \mathrm{SN}_{\mathrm{A} 53 \mathrm{~T}}(\mathbf{L})$ incubated with $5 \mathrm{mM}$ of Mimic (left) or DOPC (right) lipids. The white scale bars indicate $500 \mathrm{~nm}$.

Figure 2. Kinetic analysis of amyloid formation of aSNs in model membranes. (A-I) Maximum ThT fluorescence intensities (A, D, and $\mathbf{G})$, lag times $(\mathbf{B}, \mathbf{E}$, and $\mathbf{H})$, and elongation rate constants $(\mathbf{C}$, F, and I) of amyloidogenesis of $\alpha \mathrm{SN}_{129}(\mathbf{A}-\mathbf{C}), \alpha \mathrm{SN}_{130 \mathrm{CF}}(\mathbf{D}-\mathbf{F})$, and $\alpha \mathrm{SN}_{\mathrm{A} 53 \mathrm{~T}}(\mathbf{G}-\mathbf{I})$ in the absence and presence of Mimic (left) or DOPC membranes (right). Average values calculated from three separate samples of a single set are shown with error bars reporting the standard deviation. "n.d." denotes the concentration of lipids at which no significant increase in the ThT fluorescence intensity throughout the incubation period was observed. Various concentrations of lipids in Mimic and DOPC membranes are guided by distinct colors: black $(0 \mathrm{mM})$, light blue $(0.5 \mathrm{mM})$, blue $(1 \mathrm{mM})$, green $(2 \mathrm{mM})$, yellow $(3 \mathrm{mM})$, pink $(4 \mathrm{mM})$, and red $(5 \mathrm{mM})$. The arrows indicate a trend of changes in the lag time $(\mathbf{B}, \mathbf{E}$, and $\mathbf{H})$ and elongation rate constant $(\mathbf{C}, \mathbf{F}$, and $\mathbf{I})$.

Figure 3. Calorimetry-based characterization of interactions between $\alpha \mathrm{SNs}$ and Mimic membranes. (A-E) ITC thermograms (upper) and binding isotherms (lower) obtained by titrating $\alpha \mathrm{SN}_{w 1}(\mathbf{A}), \alpha \mathrm{SN}_{129}(\mathbf{B}), \alpha \mathrm{SN}_{130 \mathrm{CF}}(\mathbf{C})$, and $\alpha \mathrm{SN}_{\mathrm{A} 53 \mathrm{~T}}$ (D) to Mimic membranes are shown. Solid lines 
in binding isotherms indicate the fit curves based on a one-set of sites binding model. (E) Thermodynamic parameters for the binding of $\alpha \mathrm{SNs}$ to Mimic membranes.

Figure 4. Schematic models for the dual effect of Mimic membranes on $\alpha \mathrm{SN}$ amyloidogenesis. (A and B) Two models, the amyloidogenic structure model (A) and the condensation-dilution model (B) are schematically shown. Free monomers, partially- and highly-helical monomers in the membranebound forms, and amyloid fibrils are illustrated. The $N$-terminal region, the non-amyloid component region, and the $C$-terminal region of $\alpha \mathrm{SN}$ are represented in blue, grey, and red, respectively. Increases in the concentration of lipids are indicated by the purple triangle. 
bioRxiv preprint doi: https://doi.org/10.1101/2021.07.13.452016; this version posted July 18, 2021. The copyright holder for this preprint (which was not certified by peer review) is the author/funder. All rights reserved. No reuse allowed without permission.

\section{Figure 1}

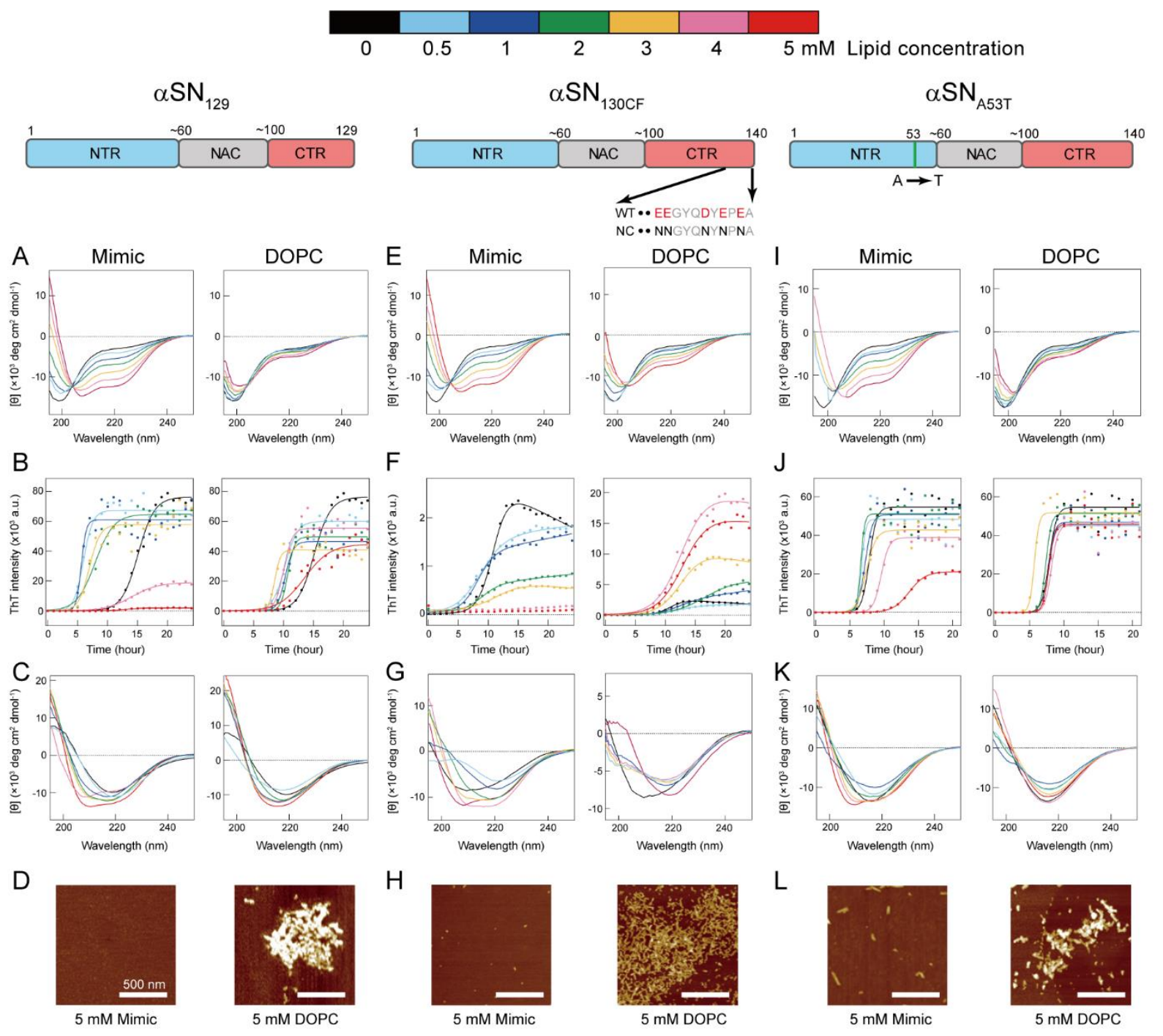


Figure 2

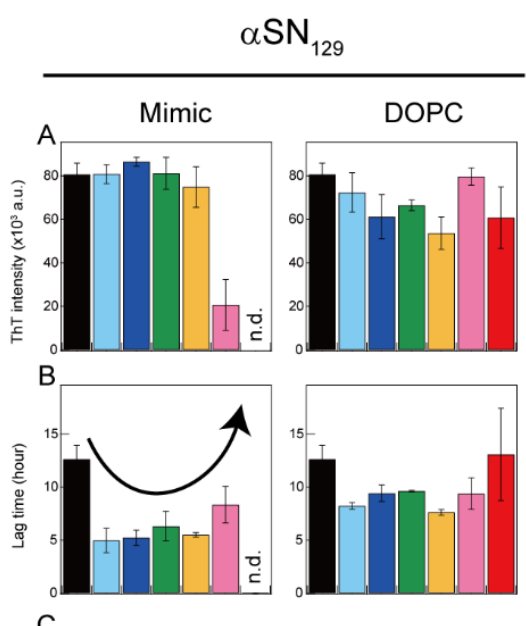

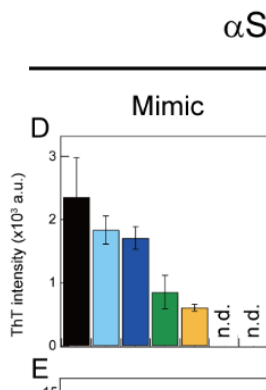

$\alpha \mathrm{SN}_{130 \mathrm{CF}}$
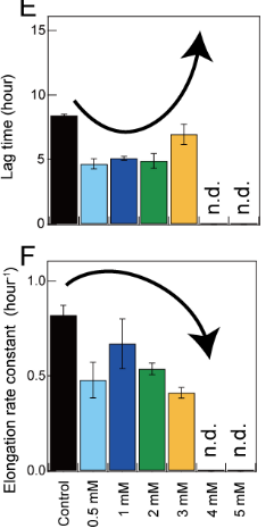

(D)

DOPC
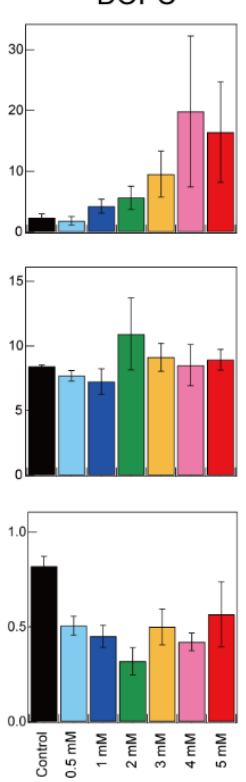
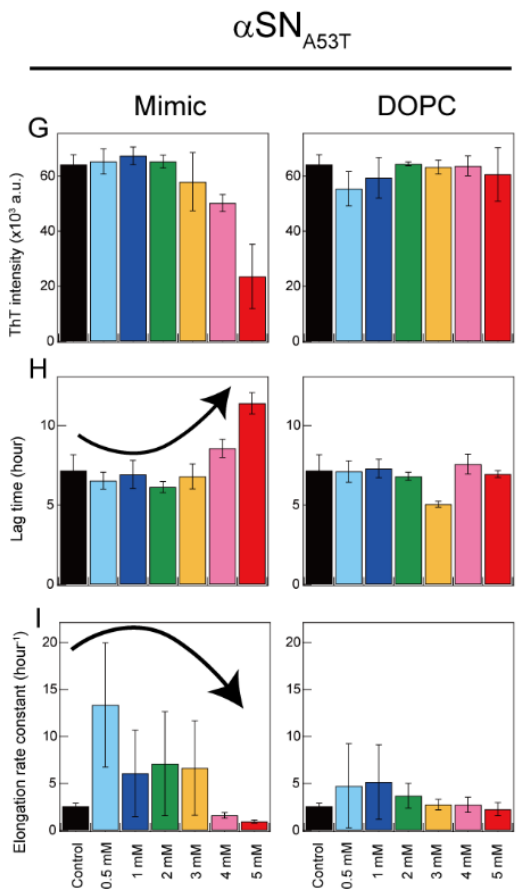
Figure 3
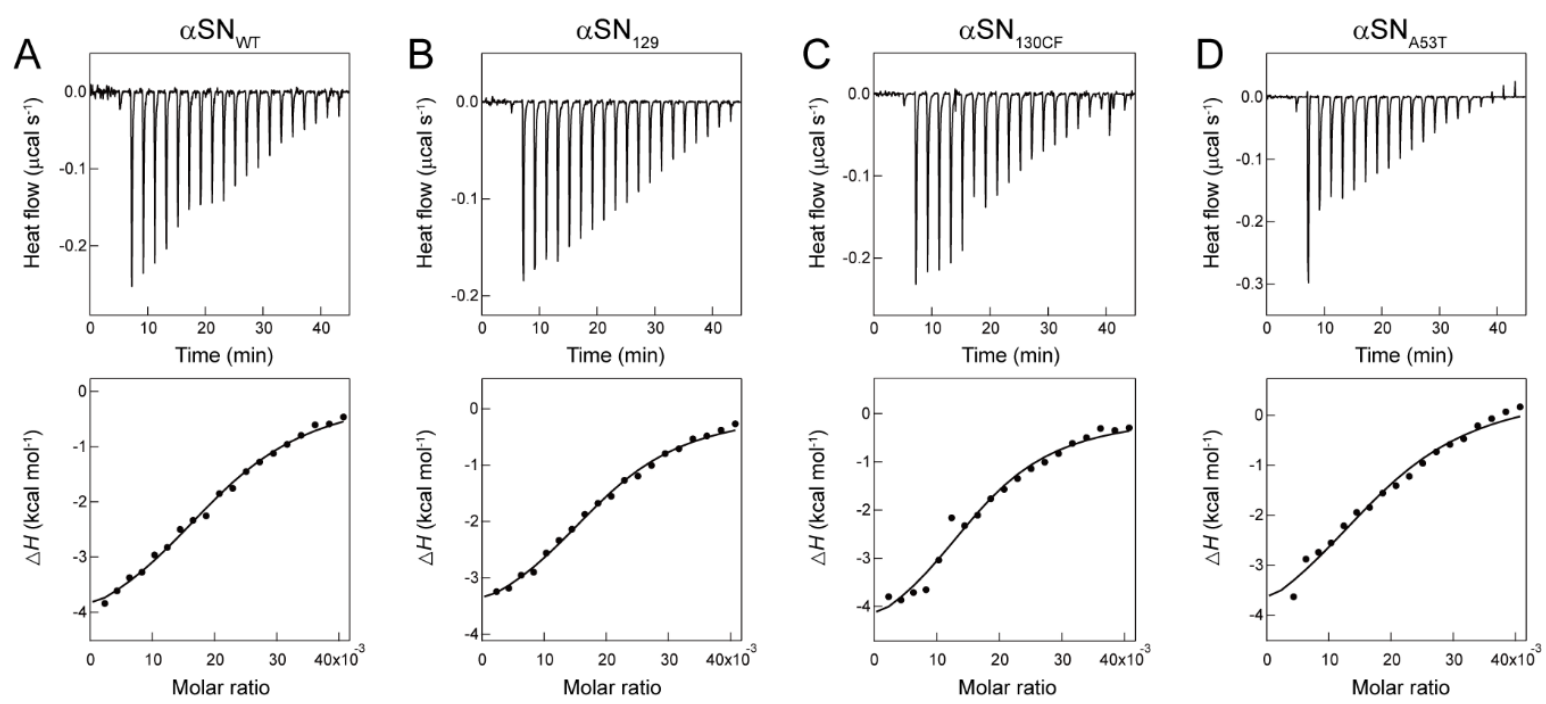

E

\begin{tabular}{|c|c|c|c|c|}
\hline & $\begin{array}{c}\Delta H \\
\left(\mathrm{kcal} \mathrm{mol}^{-1}\right)\end{array}$ & $\begin{array}{c}T \Delta S \\
\left(\mathrm{kcal} \mathrm{mol}^{-1}\right)\end{array}$ & $\begin{array}{c}\Delta G \\
\left(\mathrm{kcal} \mathrm{mol}^{-1}\right)\end{array}$ & $\begin{array}{c}K_{\mathrm{d}} \\
(\mathrm{nM})\end{array}$ \\
\hline$\alpha \mathrm{SN}_{\mathrm{WT}}$ & $-221.8 \pm 8.6$ & $-212.8 \pm 8.6$ & $-9.1 \pm 0.1$ & $197 \pm 21$ \\
\hline$\alpha \mathrm{SN}_{129}$ & $-202.5 \pm 7.4$ & $-193.4 \pm 7.4$ & $-9.3 \pm 0.1$ & $165 \pm 16$ \\
\hline$\alpha \mathrm{SN}_{130 \mathrm{CF}}$ & $-299.3 \pm 29.0$ & $-290.0 \pm 29.0$ & $-9.4 \pm 0.1$ & $134 \pm 32$ \\
\hline$\alpha \mathrm{SN}_{\text {A53T }}$ & $-287.2 \pm 33.0$ & $-278.3 \pm 33.0$ & $-9.0 \pm 0.1$ & $250 \pm 57$ \\
\hline
\end{tabular}




\section{Figure 4}

\section{A Amyloidogenic-structure model}

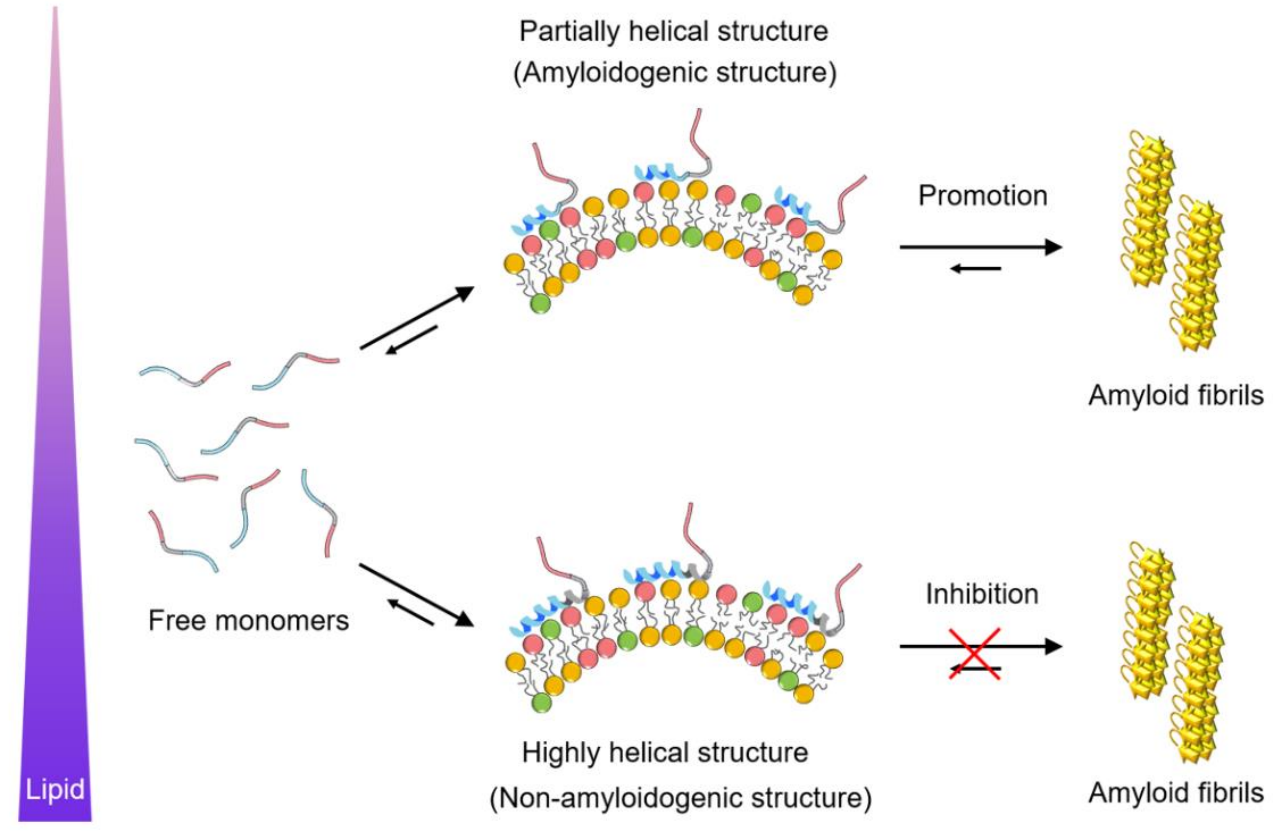

\section{B Concentration-dilution model}
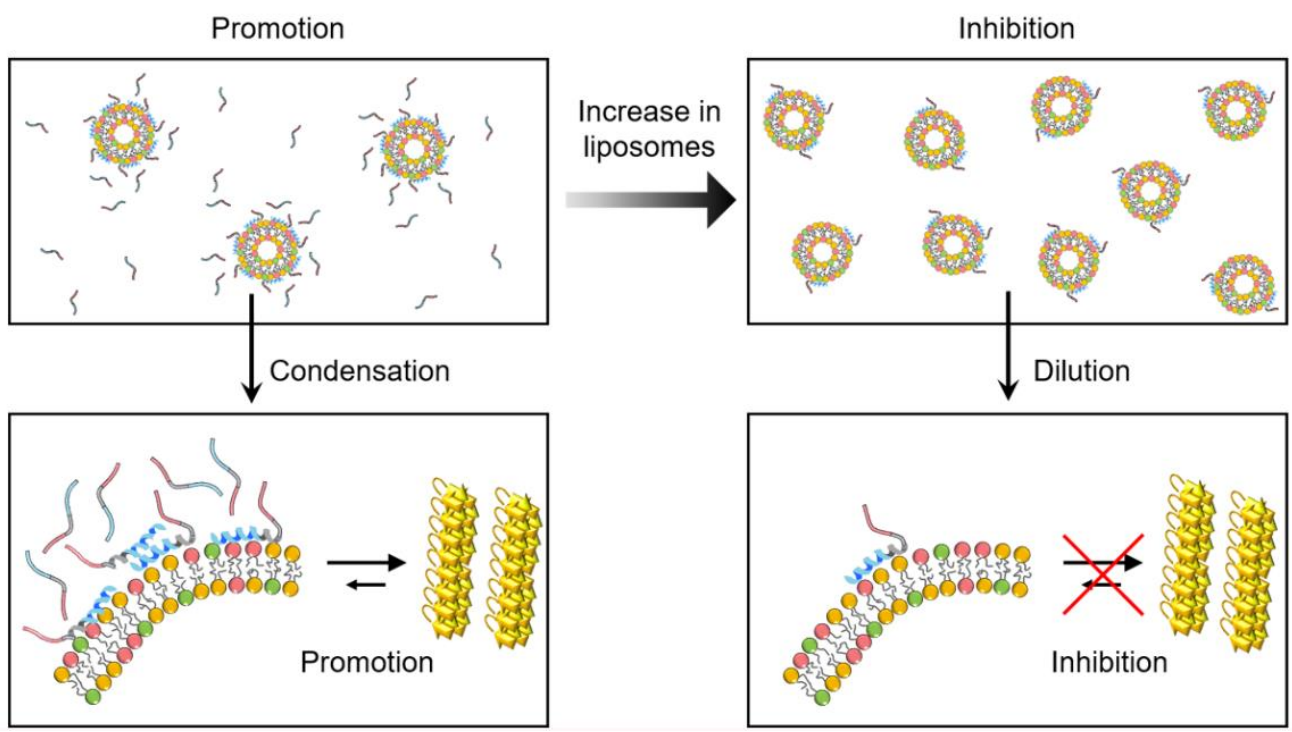